\title{
Endoscopic Ultrasound-Guided Fine-Needle Aspiration of Gastrointestinal and Pancreatic Tumors: Is Negative Pressure Helpful or Does It Suck?
}

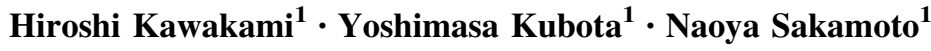

Published online: 13 October 2015

(c) Springer Science+Business Media New York 2015

Endoscopic ultrasound-guided fine-needle aspiration (EUS-FNA) facilitates endoscopic tissue acquisition. The technique is affected by two major categories of factors: that influencing sample acquisition and that influencing sample interpretation, some subject to controversy [1]. The former factors include: the degree of technical difficulty (location of the lesion), the type of lesion (solid or cystic), the diameter of the needle (19-, 22-, or 25-gauge), the number of punctures, the use of negative pressure (presence and amount including capillary aspiration, namely slow-pull technique), the presence of stylets, the presence of rapid on-site evaluation (ROSE), the type of needle (a dedicated biopsy needle, with or without a side hole at the needle tip, and with or without a core trap at the needle tip), any special maneuvers ("fanning" technique) used, and the experience and skills of the endosonographer. The factors affecting interpretation are contamination and mimicry of other types of lesions.

There is current controversy concerning the use of negative pressure suction, with regard to the decision of whether or not to use suction and the selection of an appropriate target and force of suction. In EUS-FNA, negative pressure is applied using suction with a 10- or $20-\mathrm{mL}$ syringe, which increases the quantity of the extracted sample at the expense of occasionally increasing the probability of blood contamination. In general, the outcome of a specimen gathered during EUS-FNA is determined based on the diagnostic ability of cytologist or

Hiroshi Kawakami

hiropon@med.hokudai.ac.jp

1 Department of Gastroenterology and Hepatology, Hokkaido University Hospital, Kita 14, Nishi 5, Kita-ku,

Sapporo 060-8648, Japan pathologist, acquisition of an appropriate and sufficient specimen (cellularity), contamination from the gastrointestinal (GI) tract, and observed blood contamination on slides. In the two randomized controlled trials (RCTs) that have so far evaluated the contribution of negative pressure during EUS-FNA for lymph nodes [2] or solid lesions [3], the use of negative pressure did not improve diagnostic accuracy, but did increase blood contamination. In one RCT, although the use of negative pressure increased the cellularity of the specimens obtained $(P=0.01)$, the diagnostic accuracy did not significantly differ from that of EUS-FNA without negative pressure $(P=0.73)$ [2]. Therefore, based on the available data, EUS-FNA without negative pressure is recommended by most experts [1]. If the obtained specimen is minimal, such as in cases of chronic pancreatitis, negative pressure can be used to enable the acquisition of a larger specimen [1]. Nonetheless, while most reports focus on cytology, histological tissue quality has rarely been investigated.

In this issue of Digestive Diseases and Sciences, Aadam et al. [4] hypothesized that negative pressure still persists in the aspirating needle despite closing the suction-syringe stopcock. They first examined the effect of stopcock closure and the removal of the syringe itself using an in vitro model system that confirmed the presence of residual negative pressure inside and up to the tip of the needle after closing the stopcock that was successfully neutralized by removing the syringe. In the next step, in a prospective, single-blinded, randomized, crossover pilot study in patients with solitary pancreatic masses, EUS-FNA was performed in 60 patients using a 22-gauge needle. After the first puncture and back-and-forth strokes, the syringe was either stopcock-shut but attached, or removed entirely, and a second pass was performed in a crossover fashion. The samples obtained with the syringe still connected contained 
significantly greater GI tract contamination compared to those obtained with the syringe removed (16.7 vs. $6.7 \%$, $P=0.03)$. In cases of pancreatic adenocarcinoma, the samples obtained with the syringe still connected had significantly lower cytological diagnostic yields than those obtained with the syringe removed (76 vs. $93 \%$, $P=0.02$ ). Aadam et al. concluded based on their data that neutralizing residual negative pressure by removing the syringe could improve the cytologic yield of EUS-FNA.

Negative pressure is one of the easiest and simplest factors affecting EUS-FNA outcomes [5]. Although dedicated instruments make this procedure much easier, samples are easily obtained using only syringes and extension tubes. Although it has been speculated that negative pressure is useful because it holds the tissue against the cutting edge of the needle instead of aspirating tissue into the needle [6], my group reported the utility of high negative pressure $(50 \mathrm{~mL})$ compared to customary negative pressure $(10 \mathrm{~mL})$ when using 25-gauge needles [7]. We reported significantly higher specimen acquisition rates for adequate histological diagnosis in the high negative pressure group compared to those in the normal negative pressure group (90.0 vs. $72.2 \%, P=0.0003$ ). Recently, Katanuma et al. [8] measured the actual negative pressure force and time needed to reach maximum negative pressure in different needles using a manometer in vitro. They demonstrated that the negative pressure applied to the needle increases with the diameter of the needle or the size of the syringe. Furthermore, the negative pressure generated by slowly pulling the stylet was weak. The time needed to reach maximum negative pressure was extremely long with needles with smaller diameters. Despite this understanding of the actual negative pressure and suction forces involved, the most appropriate aspiration technique is yet to be established in the clinical setting. Therefore, a RCT is warranted to confirm this hypothesis on the basis of experimental data.

To date, most previous clinical studies have been based on the diagnostic yield of cytological assessment alone during EUS-FNA, which is occasionally insufficient for diagnosing tumors of unknown origin or those with a complex background. Our RCTs demonstrated the feasibility of tissue acquisition with 22- or 25-gauge needles using a previously reported scoring system [7, 9]. In many patients, an adequate specimen is necessary in order to confirm the tumor histological subtype using immunohistochemical analysis, necessary for selecting a neoadjuvant chemotherapy regimen, especially for tumors that are borderline resectable. Cytological and histological analyses of the obtained samples should also be evaluated by adequacy scoring systems in future prospective RCTs. Aadam et al. [4] suggested that residual negative pressure may increase contamination from the GI tract, which reduces cytological diagnostic yields, especially on the first slide. Yet, the influence of GI tract contamination on histological evaluation has not been fully established. In our opinion, contamination from the GI tract and blood does not affect the accuracy of EUS-FNA when ROSE is performed by an expert cytologist or when an experienced endosonographer is available. Negative pressure during EUS-FNA takes only a few seconds and does not prolong the procedure.

In summary, Aadam et al. [4] report that despite closing the stopcock of the suction syringe, negative pressure persists in the FNA needle, not just proximal to the aspirated material but also up to the tip. Residual negative pressure contaminates the sample with unaffected GI tract tissue, which may lead to the misinterpretation of cytology specimens and thus affect cytologic yield. These results demonstrate significantly superior cytological diagnosis without residual negative pressure, which when combined with the published data provides an easy, practical, and feasible tissue acquisition technique utilizing negative pressure. Providing negative pressure during back-andforth strokes, the use of high negative pressure suction, if possible, and completely taking the syringe off the handleend is the way. Results concerning specific needle diameters and the efficacy of negative pressure are still controversial.

In conclusion, the careful application of negative pressure may increase diagnostic yield for EUS-FNA - guided sampling of solid pancreatic tumors. The details of the technique with respect to the amount of pressure applied, needle size, and duration of the negative pressure among other factors are subject to confirmation by additional RCTs.

\section{Compliance with ethical standards}

Conflict of interest Dr. Kawakami is a consultant and gives lectures for the Olympus Corporation, Tokyo, Japan, Piolax Medical Devices, Kanagawa, Japan, and Taewoong-Medical Co., Ltd., Seoul, South Korea. Dr. Kawakami is a consultant for Zeon Medical Inc., Tokyo, Japan, and M.I.Tech Co., Ltd., Seoul, South Korea. No potential conflicts of interest relevant to this article were reported.

\section{References}

1. Varadarajulu S, Fockens P, Hawes RH. Best practices in endoscopic ultrasound-guided fine-needle aspiration. Clin Gastroenterol Hepatol. 2012;10:697-703.

2. Wallace MB, Kennedy $\mathrm{T}$, Durkalski V, et al. Randomized controlled trial of EUS-guided fine needle aspiration techniques for the detection of malignant lymphadenopathy. Gastrointest Endosc. 2001;54:441-447.

3. Puri R, Vilmann P, Săftoiu A, et al. Randomized controlled trial of endoscopic ultrasound-guided fine-needle sampling with or without suction for better cytological diagnosis. Scand J Gastroenterol. 2009;44:499-504. 
4. Aadam A, Oh YS, Shidham VB, et al. Eliminating the residual negative pressure in the endoscopic ultrasound aspirating needle enhances cytology yield of pancreas masses. Dig Dis Sci. (Epub ahead of print). doi:10.1007/s10620-015-3860-0.

5. Lee JK, Choi JH, Lee KH, et al. A prospective, comparative trial to optimize sampling techniques in EUS-guided FNA of solid pancreatic masses. Gastrointest Endosc. 2013;77:745-751.

6. Savides TJ. Tricks for improving EUS-FNA accuracy and maximizing cellular yield. Gastrointest Endosc. 2009;69:S130 S133.

7. Kudo T, Kawakami H, Hayashi T, et al. High and low negative pressure suction techniques in EUS-guided fine-needle tissue acquisition by using 25-gauge needles: a multicenter, prospective, randomized, controlled trial. Gastrointest Endosc. 2014;80:10307.e1.

8. Katanuma A, Itoi T, Baron TH, et al. Bench-top testing of suction forces generated through endoscopic ultrasound-guided aspiration needles. J Hepatobiliary Pancreat Sci. 2015;22:379-385.

9. Abe Y, Kawakami H, Oba K, et al. Effect of a stylet on a histological specimen in EUS-guided fine-needle tissue acquisition by using 22-gauge needles: A multicenter, prospective, randomized, controlled trial. Gastrointest Endosc. 2015. doi:10.1016/j.gie. 2015.03.1898 